\title{
Choice blindness and choice-induced preference change in groups
}

\author{
Philip Pärnamets* \\ Department of Clinical Neuroscience, Karolinska Institutet, \\ Sweden. \\ Department of Psychology, New York University, USA. \\ Cognitive Science, Lund University, Sweden. \\ Gabriel Vogel \\ Lars Hall \\ Cognitive Science, Lund University, Sweden.
}

\author{
Jorina von Zimmermann \\ Ramsey Raafat \\ University College London, University of London, UK.
}

\author{
Petter Johansson \\ Cognitive Science, Lund University, Sweden.
}

\section{This is a pre-print and it has not yet undergone peer-review.}

\begin{abstract}
Contrary to common belief, our preferences do not only shape our decisions but are also shaped by what decisions we make. This effect, known as choice-induced preference change, has been extensively studied in individuals. Here we document choice-induced preference change in groups. We do so by using the choice-blindness paradigm, a method by which participants are given false feedback about their past choices. Participants are given a second round of choices following the choice blindness manipulation'measuring preference change resulting from accepting the manipulation. In Experiment $1(\mathrm{~N}=83)$, we introduce a roommate selection task used in this paper and use it to replicate choice-induced preference change using choiceblindness in individuals. In Experiment $2(\mathrm{~N}=160)$, dyad members made mutual choices in the roommate selection task and then receive either veridical or false feedback about what choice they made. The majority of the false feedback trials were accepted by the dyads as their own choices, thereby demonstrating choice blindness in dyads for the first time. Dyads exhibited choice-induced preference change and were more likely to choose the originally rejected option on trials where they accepted the manipulation compared to control trials. In Experiment 3 $(\mathrm{N}=80)$, we show that the preference effect induced by the choice blindness manipulation at the group level does not generalize back to follow up choices made by individual participants when removed from the group. In all studies, response time analyses further support our conclusions. Choice-induced preference change exists for both individuals and groups, but the level at which the choice was made constrains the influence of that choice on later preferences.
\end{abstract}

Keywords: choice blindness, decision making, preference change, group decisions, self-perception, cognitive dissonance

Few findings have been as influential in the history of psychology as the discovery of choice-induced preference change - that choices not only reflect an individual's preferences but also shape them (Bem, 1972; Brehm, 1956; Chen \& Risen, 2010; Egan, Bloom, \& Santos, 2010; Festinger, 1962; Gerard \& White, 1983; Harmon-Jones \& Mills, 2019; Izuma \& Murayama, 2013). Part of this influence stems from how it contradicts our common sense understanding of our choices and their relation to our desires and beliefs. Choiceinduced preference change has also been theoretically influential, both for the development of cognitive dissonance theory (Brehm, 1956; Festinger, 1962) and as a challenge to classical decision theory in which choices almost by definition have been thought to follow preferences (Edwards, 1954; Slovic, Fischhoff, \& Lichtenstein, 1977). Moreover, many of life's decisions are not made by individuals by themselves but by individuals within and embedded in groups. The processes involved in group decision making have been extensively investigated, including the steps leading up to how decisions are reached (Kerr \& Tindale, 2004; Kugler, Kausel, \& Kocher, 2012), and how group members' preferences align (Cialdini \& Goldstein, 2004; Raafat, Chater, \& Frith, 2009). But what happens when the group is asked to make a second choice - will the previous decision shape 
the present one analogous to the individual case? Using the choice blindness paradigm - a novel methodological tool in the study of choice-induced preference change - we test if choice-induced preference change also occurs in the smallest possible group structure, the dyad.

\section{Choice-induced preference change}

Choice-induced preference change was originally introduced with the free choice paradigm (Brehm, 1956). In the free choice paradigm participants first rate their preference for a number of options, then make choices between pairs of options closely matched in rated preference. Finally, participants rate the options a second time post-choice. The key finding, indicating choice-induced preference change, is that the chosen option is rated higher and the non-chosen lower than previously, resulting in a "spreading" of the preference distance between the two options. Choice-induced preference change challenges a core assumption about the relationship between preferences and decisions. In standard economic and psychological decision models, choices are assumed to result from decision-makers' preferences (Edwards, 1954; Slovic et al., 1977), and these preferences should only change if new information is gained about the choice options. But nothing new is added in the mere act of selecting one of the options, which indicates the presence of some form of feedback loop between decision outcomes and valuation.

The free choice paradigm was introduced to test a prediction from cognitive dissonance theory (Brehm, 1956; Festinger, 1962; Harmon-Jones \& Mills, 2019), and is usually invoked as evidence in support of that theory. According to cognitive dissonance theory, the act of choosing between two similarly valued options induces a conflict in the decisionmaker following the choice. The source of this conflict is the cognitive inconsistency of previously having entertained the positive aspects of the non-chosen alternative and the nega-

Author contributions: P.P., P.J, L.H \& N.C. developed the study concept and all authors contributed to the study design. J.vZ. G.V.\& P.P performed testing and data collection. P.P. performed the data analysis. P.P. \& P.J. drafted the manuscript and all authors provided critical revisions and approved the final version of the manuscript for submission.

Funding information: N.C. was supported by ERC grant 295917-RATIONALITY, the ESRC Network for Integrated Behavioural Science [grant number ES/K002201/1], the Leverhulme Trust [grant number RP2012-V-022], Research Councils UK Grant EP/K039830/1, and the Templeton Foundation. P.J. and L.H. was supported by the Swedish Research Council (2014-1371). P.P was supported by Swedish Research Council (2016-06793).

Conflicting interests: The authors report no conflicts of interest.

*Corresponding author: philip.parnamets@ki.se tive aspects of the selected alternative. This experience of dissonance may be reduced by changing ones preferences so as to make the chosen alternative more desirable, and the non-chosen option less desirable, than they were before the choice. By contrast, self-perception theory (Bem, 1972) explains choice-induced preference change without recourse to conflicting internal states. Instead, the theory posits that the decision-maker infers that their preferences for one option is likely larger, given that they just choose it, in the same way they would have inferred it if observing another decision maker.

While the competing frameworks of self-perception theory and cognitive dissonance theory are notoriously difficult to adjudicate between, one thing they have in common is that the mechanisms they posit operate at an individual level. But what would happen if a group makes a collective decision, would the group's preference then also change as a result? And if so, what consequences would it have for the preferences of the constituent individuals? For example, an individual as a member of group can influence the preferences of that group, and hence is expected to be consistent with the decisions of the group in their later choices. But the group's decision may also present an independent influence on constituent individuals' preferences, also driving consistency with the group choice. These causal questions are difficult to disentangle experimentally. One way to circumvent this difficulty is to dissociate the link between the dyad members' initial preferences and the outcome of their choice. To do so, we turn to the choice blindness paradigm, which we introduce next.

\section{Choice blindness and preference change}

The choice blindness paradigm, originally introduced to study self-knowledge about one's own choices (Johansson, Hall, Sikström, \& Olsson, 2005), involves giving participants false feedback about their choices through sleight-of-hand or other forms of manipulation. For example, in Johansson et al. (2005), participants chose which of two presented faces was most attractive to them. Following their choice, their selection was re-presented to them and participants were asked to state the reasons for their choice. Unknown to them, on certain trials, a double-card conjuring trick was used to covertly exchange one face for the other. On the great majority of trials, participants failed to notice the mismatch between choice and outcome, while still being prepared to offer introspective reasons for why they chose the way they thought they had. Choice blindness is not limited to attractiveness decisions but has been extended to moral and political attitudes (Hall, Johansson, \& Strandberg, 2012; Strandberg, Sivén, Hall, Johansson, \& Pärnamets, 2018), eye-witness identification (Sagana, Sauerland, \& Merckelbach, 2014), memory reports (Cochran, Greenspan, Bogart, \& Loftus, 2016), as well as economic and consumer decisions (Cheung et al., 2016; 
McLaughlin \& Somerville, 2013).

Results from the choice blindness paradigm not only show that participants often fail to correct the manipulation and confabulate reasons for making a choice they in fact never made, but also that accepting the false feedback has downstream consequences on participants' cognition. In Johansson, Hall, Tärning, Sikström, and Chater (2014), participants made preferential choices between pairs of faces, as described previously. However, in this experiment, following the first round of choices, participants were also asked to take part in a second round of choices using the same face pairs. The pattern found was that in manipulated trials, and particularly for accepted manipulations, the initially rejected faces were chosen more frequently in the second round. This is a clear demonstration of a choice-induced preference change, as the participants come to prefer the alternative they were led to believe they had previously chosen.

In this situation, it is also clear that it cannot be the decision process itself which affects the later preference, but rather the belief about a previous choice. In support of this interpretation, research has shown that participants form false memories of past choices following acceptance of the manipulation in the choice blindness paradigm (Pärnamets, Hall, \& Johansson, 2015). Furthermore, choice-induced preference change in the choice blindness paradigm is replicable (Izuma et al., 2015; Luo \& Yu, 2017; Taya, Gupta, Farber, \& Mullette-Gillman, 2014), and extends to manipulations of political attitudes with effects lasting a week post manipulation (Strandberg et al., 2018).

In sum, the choice blindness paradigm provides a robust method to study choice-induced preference change. In the present study we used the choice blindness paradigm to study choice-induced preference change in dyads. We let two people make a joint decision and sometimes manipulated the outcome of their choices. We measured to what extent they correct the manipulation, and if accepting the manipulation led to preference change as measured in subsequent choices. The novel factor of false-feedback at the group level introduces an unknown element into the choice blindness paradigm. To date, no studies employing this paradigm have been performed on groups, and it is therefore unknown, not only if there will be a preference change effect in such a set-up, but also whether moving into the social domain of groups will impede, dilute or indeed remove any susceptibility to the choice blindness manipulation in the first place. Discovering that choice blindness transfers from individuals to groups opens up avenues for new research on groups and choices, including the question investigated here if choiceinduced preference change affects groups decisions.

\section{Group decisions and performance}

Our study is an attempt to use the choice blindness paradigm to study choice-induced preference change in a group setting. As the mechanisms proposed for cognitive dissonance and self-perception theory operate at the individual level, we turn to the literature on group performance and group decision making to garner predictions for what may occur in our task.

On the one hand, groups may be less successful in correcting the choice blindness manipulation as individual members may be prone to social loafing (Ingham, Levinger, Graves, \& Peckham, 1974), possibly brought about by a diminished sense of shared responsibility over the decision (Weldon \& Gargano, 1985). On the other hand, there are several reasons to believe that groups may be more successful at detecting the choice blindness manipulation compared to individual participants. Social interaction during deliberation may promote processing at a deeper level, making correction more likely through enhanced memory performance (Lejarraga, Lejarraga, \& Gonzalez, 2014; Olsson, Juslin, \& Olsson, 2006; Shteynberg, 2015). Compared to individuals, groups are also better at visual search (Brennan, Chen, Dickinson, Neider, \& Zelinsky, 2008; Malcolmson, Reynolds, \& Smilek, 2007a, 2007b). In line with this, dyads have been found to outperform individuals in standard change blindness experiments, but only if they are allowed to communicate during the task (Knott, Nelson, McCroskey, \& Miller, 2007). Correcting the choice blindness manipulation can be characterized as one of independent aggregation of judgments, a case where groups typically outperform individuals through 'wisdom of the crowds' effects (Tindale \& Winget, 2019). In sum, we expect higher correction rates for groups compared to individuals, and we cannot a priori discount the possibility that correction levels will be so high so as to make it difficult to study preference effects in groups using it.

If groups are susceptible to the choice blindness manipulation, we hypothesize, based on previous work on choice blindness and preference change (Johansson et al., 2014; Luo \& Yu, 2017; Strandberg et al., 2018; Taya et al., 2014), that the dyads will also exhibit preferential shifts. This is a far from trivial prediction. A key difference to individual decision-making is the necessarily public nature of deliberation preceding group choices. The group members, to form a mutual decision, will need to discuss and argue for the different options. In our task, where participants chose between one of two persons as a potential future roommate, this means that both visual features of the chosen person as well as other reasons underpinning the group's choice will be highlighted and made public to all members of the group. Additionally, groups have been found to have superior memory to individuals in decision tasks (Lejarraga et al., 2014; Olsson et al., 2006). Likewise, individual members of a group can calibrate to one another as well as correct each other's mistakes (Bahrami et al., 2010). These two characteristics ought to enforce preferential consistency with the original choice. Nevertheless, if the choice blindness manip- 
ulation is accepted and the members of the dyad shift their beliefs about their past choice, the same mechanisms of consistency may work to enhance the preference effect.

\section{Overview of present study}

We report the results from three experiments. First, we adapt the standard choice blindness task (Johansson et al., 2005), in which participants make preferential choices based on attractiveness. While beauty norms can be shared, they are also a domain where group deliberation is not commonplace and there is considerable room for locked disagreements. In the current experiment, we therefore let participants look at pairs of faces and decide who they think would make the best roommate, a domain where our participants, being college students, ought to have some experience and be in possession of a common language for negotiating a mutual decision. Participants then complete a second round of choices allowing us to measure choice-induced preference change. In experiment 1 we test this novel task on individuals, effectively creating a baseline to compare both correction and preference change rates in groups against.

In our second and main experiment, we had pairs of participants make mutual decisions in the roommate task and gave the dyad false feedback about their choices on some trials, before letting the dyad do a repeated set of choices. Doing so gave us opportunity to test if choice blindness and choice-induced preference change extends to groups. In Experiment 3 we again have pairs of participants undergo the choice blindness manipulation, but they make their second round choices individually. This allowed us to investigate what happens to individual preferences following a manipulation of the group preference. In addition, we investigated second round choice response times. Choice response times have a long history in psychology for inferences about cognitive processes and strength of preference (Konovalov \& Krajbich, 2019; Link, 1975; Ratcliff \& Rouder, 1998; Tyebjee, 1979). However, little is known about how false-feedback might affect follow-up choice response times in the choice blindness paradigm. By comparing choice response times in corrected and accepted manipulated trials with control trials we aimed to gauge similarities or differences in how choices are made and to what extent the false-feedback is integrated into participants' preferences.

\section{Experiment 1: Individuals in the roommate selection task}

\section{Method}

Participants. A total of 83 participants were recruited from the student populations at University College London and Lund University. Sample size was selected to be double that of Johansson et al. (2014). Average age was 24.6 $(S D=5.9)$. 25 participants were male. All participants received either course credit or a payment of $£ 3$ (UCL) or a cinema voucher (Lund) for participation. Experiment 1 was approved by UCL Departmental Ethics Committee (CPB/2010/006) and the Ethics Committee of Lund University (D.nr. 2016-1046).

Materials and procedure. Participants undertook a novel computerized CB task, programmed in PsychoPy (Peirce, 2007) ${ }^{1}$, during which they were asked to imagine that they shared an apartment with a spare room, and were now looking for a new female roommate. We presented the participants with twenty pairs of pictures of female faces, and they had to decide who in each pair they would rather live with. Participants were video-taped throughout the experiment to allow classification of manipulated trials after the experiment had completed. Each face pair was presented on virtual playing cards for $2 \mathrm{~s}$ on the screen, before the cards flipped over to conceal the faces. The participant selected their preferred candidate by button press (see Fig. 1). After participants had chosen, they completed a seven-point Likert scale indicating how high their individual confidence was in their choice. This provides a natural occlusion time between the original choice and the manipulation which is a crucial feature of the choice blindness paradigm. Finally, we presented the chosen face a second time to the participants. During this second presentation, an onscreen question prompted participants to discuss their reasons for choosing the person they had selected, and specifically to do so in the context of a personality trait rating presented onscreen. The traits prompted were from the list of: reliable, desirable, friendly, humorous, and, easy-going. To ensure that the participants attended the question and thus the chosen face during the second presentation, these trait options were randomly distributed across trials. The chosen face was visible throughout the participants' explanation until they completed the rating.

On four trials, out of the total of 20 trials, we surreptitiously altered the participants' choices, so that they received false feedback about their selection and the rejected candidate was presented to them as the preferred one (hereafter manipulated trials). For the manipulation, we used eight possible 'target' pairs, positioned in the following trial pairs: $<5,6>,<9,10\rangle,<13,14>$ and $<18,19>$ (see Supplementary Figure 1). At the start of each experiment we randomly selected four of these trials, one from each trial pair, to be manipulated, while the four non-selected trials were used as non-manipulated control stimuli in the subsequent analyses.

Following the twenty initial choices, a second, previously unannounced choice phase commenced in which the eight target pairs were presented a second time in a random order. We asked participants to make a second choice between the candidates in each pair. Participants were asked to imagine that the pool of available candidates had reduced

\footnotetext{
${ }^{1}$ Code to run experiment available at: https://osf.io/ y6hkx/
} 

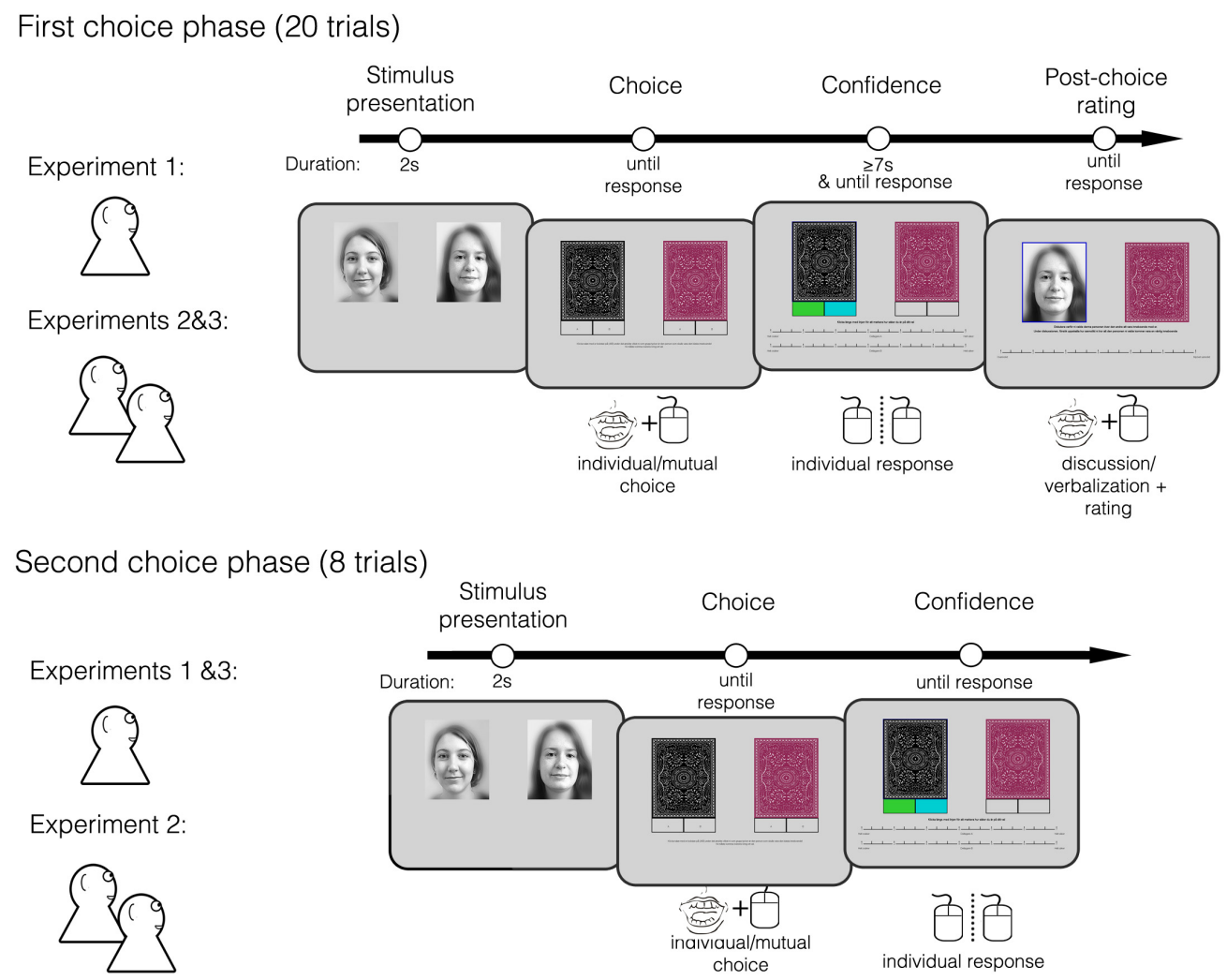

Figure 1. Overview of experimental procedure. First choice phase. The first choice phase consisted of 20 trials. Participants viewed candidates presented pairwise for $2 \mathrm{~s}$. After viewing they selected their preferred candidate by clicking the choice box beneath the card where the candidate had been presented. Participants then indicated their confidence in their choice. Finally their chosen candidate was represented to them and participants were asked to discuss and rate one personality feature of that candidate. In 4 trials (manipulated trials) the non-chosen candidate was presented instead of the chosen one (pictured). In Experiments $2 \& 3$ (pictured) participants were dyads, who had to come to a mutual agreement to make their choices. Second choice phase. A second choice phase followed the first. Participants were asked to make choices and give confidence ratings for 8 target pairs - the four manipulated and four non-manipulated. In Experiments $1 \& 3$ the phase was completed individually while in Experiment 2 participants remained in their dyad and continued to make mutually agreed decisions.

and simply once again choose whomever they thought would make the best roommate. All participants, therefore, chose between 4 previously manipulated pairs and 4 control pairs which had not been manipulated previously. Their previous choices were not indicated to the participants and no further manipulations were performed during this phase. Following each choice, the participant indicated their confidence in their choice, after which the next trial commenced.

At the end of the experiment participants were fully debriefed, signed informed consent, rewarded, and thanked for their participation.

Statistical analysis. All analyses were performed in the $\mathrm{R}$ statistical language using the brms package (Bürkner et al., 2017). We analyzed the data using Bayesian multi-level regression. All analyses included varying intercepts and slopes grouped by participant ID and by stimulus ID, as well as, correlations between group varying intercepts and slopes.
For all analyses we report the the parameter estimate, the 95\% Highest Density Interval (HDI) and probability of direction (pd; proportion of posterior in the region sharing sign with the parameter estimate). HDI and probability of direction were calculated using the bayestest $R$ package (Makowski, Ben-Shachar, \& Lüdecke, 2019). We additionally report Savage-Dickey Bayes Factors to quantify evidence of an effect and interpret Bayes Factors $>=3$ as weak evidence for an effect and $>=10$ as strong evidence for an effect.

We report analyses on participants' correction of the manipulation as well as their second round choices and response times. See the Supplementary Materials for analyses involving confidence.

Data and code availability. Experimental data and analysis code for this and all following experiments are available from the Open Science Framework https://osf.io/ 
y6hkx/

\section{Results}

Correction of manipulation. Using the audio and video recordings as evidence, a manipulated trial was classified as having been corrected if the participants voiced suspicion that a switch of pictures had taken place and as a result clearly questioned that their chosen picture was the one being displayed.

Of the 332 manipulated trials, 78 (23\%) were corrected, $253(76 \%)$ were accepted and 1 trial was not classified due to equipment malfunction. 41 (49\%) participants failed to make any corrections, while on average each participant made 0.95 corrections $(S D=1.19)$.

Choice consistency. We defined choice consistency as congruence between first and second choice. Participants made second round choices consistent with their original decision in $83.4 \%$ of control trials. For corrected manipulated trials, consistency was higher, $94.9 \%$, while for accepted manipulated trials consistency was lower $70.0 \%$, see Fig $2 \mathrm{~A}$. We analyzed second round choices using mixed-effects logistic regression, taking control trials as the reference level and adjusting for first round choice confidence.

In line with our hypothesis, we found strong evidence that participants were less consistent when accepting the manipulation, both compared to non-manipulated control trials ( $b$ $=-0.82, S E=0.28, H D I(95 \%$ Highest Density Interval $)=$ $[-1.36,-0.26], p d$ (probability of direction) $=0.9976, B F_{10}$ $($ Bayes Factor $)=12.7)$ and compared to corrected trials $(b$ $=-2.11, S E=0.55, H D I=[-3.21,1.08], p d \approx 1.0, B F_{10}=$ 732). Participants were also more consistent in choices following corrected trials compared to control trials $(b=1.29$, $\left.S E=0.54, H D I=[0.26,2.38], p d=0.9956, B F_{10}=10.82\right)$. Overall the model indicated only an anectodal effect of first round confidence on subsequent consistency $(b=0.37, S E=$ $\left.0.18, H D I=[0.00,0.74], p d=0.9722, B F_{10}=1.29\right)$, and there was no evidence for any differences by trial type in this regard (all $B F s_{10}<0.35$ ).

Second round choice response times. We analyzed choice response times to the second presentation of the faces (i.e. when consistency is measured) split by trial type and if the choice was consistent or not. By comparing the manipulated trials against control trials we explored the degree inconsistent choices in accepted trials shared looked like consistent choices in general. For control trials (consistent: $M=$ $1.2 \mathrm{~s}, S D=0.9$, inconsistent: $M=2.3 \mathrm{~s}, S D=3.5$ ) and corrected trials (consistent: $M=1.0 \mathrm{~s}, S D=1.0$, inconsistent: $M$ $=3.5 \mathrm{~s}, S D=2.3$ ) a pattern emerged where participants were considerably slower on inconsistent trials than on consistent trials (see Fig 2B). By contrast on accepted trials participants had similar choice response times when they were consistent $(M=1.6 \mathrm{~s}, S D=1.6)$ as when they were inconsistent $(M=$ $1.4 \mathrm{~s}, S D=1.4)$.
To test these patterns statistically we performed a mixedeffects regression on the log-transformed choice response times. We found evidence for a difference in choice response times between consistent and inconsistent choices, such that inconsistent choices were on average slower than inconsistent choices $(b=0.55, S E=0.17, H D I=[0.22,0.90], p d$ $\left.=0.9983, B F_{10}=23.13\right)$. On average, there were no differences in choice response times between trial types (all $B F s_{10}$ $<0.35$ ). We next computed contrasts between consistent and inconsistent choices separately for each trial type. We found evidence for inconsistent choices being slower than consistent choices for both control trials $(b=0.47, S E=0.20, H D I$ $\left.=[0.09,0.87], p d=0.9930, B F_{10}=4.88\right)$ as well as for corrected trials $(b=1.14, S E=0.40, H D I=[0.33,1.91], p d$ $\left.=0.9972, B F_{10}=23.09\right)$. Critically, for accepted trials we instead found evidence favoring the null of no difference $(b$ $=0.039, S E=0.18, H D I=[-0.32,0.41], p d=0.59, B F_{10}$ $=0.197)^{2}$. In sum, for control and corrected trials there is a choice response time difference between consistent and inconsistent choices, while for accepted trials there is no such difference.

\section{Summary Experiment 1}

We introduced the roommate selection task where participants chose between pairs of faces presented to them who they would rather have as a roommate. Participants had no problem accepting and performing the task as intended, and were able to make choices as well as verbalize reasons for their decisions. On some trials we gave false feedback to participants about who they had selected and found, consistent with the predictions of previous choice blindness research (Johansson et al., 2005, 2014; Pärnamets et al., 2015), that participants accepted the manipulation in the majority of trials further extending the choice blindness paradigm into this domain of choices.

On trials where participants accepted the manipulation, participants were also more likely to switch their preference in a second round of choices. This choice-induced preference change was specific to accepted trials, consistent with recent findings from manipulating political attitudes in the choice blindness paradigm (Strandberg et al., 2018). More generally, these findings replicate previous work on preference change following choice blindness, testifying to the robustness of the paradigm in this context (Johansson et al., 2014; Luo \& Yu, 2017; Strandberg et al., 2018; Taya et al., 2014).

We also explored the second round choice response times and found a striking pattern of results. For both control and corrected trials inconsistent round two choices were considerably slower compared to consistent choices. In other words, changing one's mind takes longer time than to reaf-

\footnotetext{
${ }^{2} B F_{01}=5.06$
} 


\section{Experiment 1: Individuals}
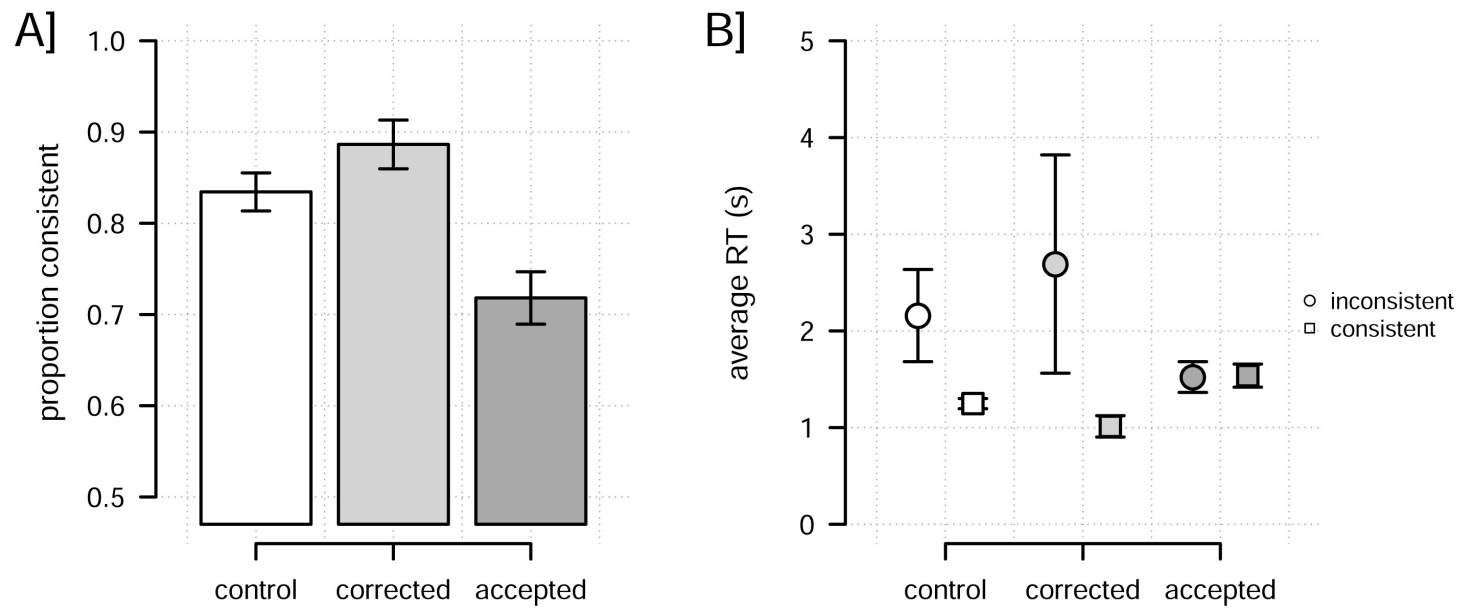

Figure 2. A] Proportion second round choices consistent with first round choices in Experiment 1. Error bars indicate standard errors. B] Average choice response times for second round choices in Experiment 1, split by consistency with first round choices. Circles represent inconsistent choices and squares consistent choices. Error bars indicate standard errors.

firm a previously made decision. However, for the accepted manipulated trials the choice response time difference between consistent and inconsistent second round choices disappeared almost completely. Following an acceptance of the manipulation, inconsistent choices - where preference change is observed - are not changes of mind but rather represent an affirmation of a false belief about the original preference (cf. Pärnamets et al., 2015). This is further evidence that the participant has fully integrated the accepted manipulated choice.

\section{Experiment 2: Preference change in groups}

Having established the roommate selection task, we turned to our main question - investigating choice-induced preference change in groups. In Experiment 2 we tested if groups are susceptible to the choice blindness manipulation, and if accepting the false feedback would similarly lead to preference changes in a second round of choices.

\section{Method}

\section{Participants}

A total of 160 participants (80 dyads) were recruited from the student populations at University College London, Malmö University and Lund University and of these 152 participants (76 dyads) were successfully recorded. Average age was $24.9(S D=5.3)$. 63 participants were male. 35 dyads were mixed, 27 were female only, and 14 male only. All participants received $£ 4$ (UCL) or a cinema voucher (Malmö/Lund) for participation. Dyads were formed by participants signing up to time slots we made available through an online booking system. Before participating, we vetted the dyads to make sure that members were not acquainted with each other. Experiment 2 was approved by UCL Departmental Ethics Committee (CPB/2010/006) and the Ethics Committee of Lund University (D.nr. 2016-1046).

Procedure. Procedure was identical to Experiment 1 with minor changes to accommodate dyads instead of individuals. Participants were left on their own in the experiment room at the start of experiment, and their interaction was solely captured by audio and video. Roommate selection, both in the first and the second round, was a mutual decision. We told the participants that they had to discuss and agree on a choice, but no instructions were given as to how they should reach an agreement. After the participants had chosen, they each completed a seven-point Likert scale indicating how high their individual confidence was in their mutual choice, in effect measuring how much they agreed with the group choice. When presented with their choice a second time - when on manipulated trials false feedback was given participants were instructed to discuss with one another why they had chosen the option they did, and in the context of this discussion to rate how reliable, desirable, friendly, humorous, or easy going they thought the chosen candidate would be. See also Fig. 1.

\section{Results}

Correction of manipulation. Using the audio and video recordings as evidence, a manipulated trial was classified as having been detected if at least one of the participants voiced suspicion that a switch of pictures had taken place and if as a consequence both participants came to agree that the picture they were seeing was not the one they had originally chosen. 
Of the 304 M-trials across the 76 dyads, 107 (36\%) were corrected, $194(64 \%)$ were accepted and 3 trials were not classified due to equipment malfunction. 30 (39\%) dyads failed to make any correction, while on average each dyad made 1.41 corrections $(S D=1.38)$.

The correction rates are higher in Experiment 2 than in Experiment 1. However, our correction rates appear consistent with the individual corrections rates combining to provide the correction rates for dyads. If two individuals in Experiment 1 are considered separately, then the chance that neither correct the manipulation is $0.76 * 0.76=0.58$. The number of trials not detected by the dyads in Experiment 2 was very close to this value (0.64). Thus, choice blindness appears to extend linearly from individuals to dyads.

Choice consistency. Participants made second round choices consistent with their original decision in $85.9 \%$ of control trials. For corrected manipulated trials consistency was similar, $81.3 \%$, while for accepted manipulated trials consistency was lower $62.9 \%$, see Fig 3A. We analyzed second round choices using mixed-effects logistic regression, taking control trials as the reference level. To adjust for confidence in first round choices, total confidence (i.e. the sum of both participants' ratings) was used.

As hypothesized, we found strong evidence that participants were less consistent having accepted the manipulation compared to control trials $(b=-1.40, S E=0.40, H D I=[-$ 2.21, 0.63], $\left.p d=0.9992, B F_{10}=66.79\right)$ and weak evidence when compared to corrected trials $(b=-1.06, S E=0.47, H D I$ $\left.=[-0.60,1.31], p d=0.9873, B F_{10}=4.87\right)$. We found no evidence for a difference in consistency comparing control trials to corrected trials $(b=0.35, S E=0.49, H D I=[-0.60$, $\left.1.31], p d=0.7726, B F_{10}=0.45\right)$. Unlike Experiment 1, the model indicated weak evidence for an effect of first round confidence on subsequent consistency $(b=0.52, S E=0.20$, $\left.H D I=[0.12,0.94], p d=0.9918, B F_{10}=5.04\right)$. However, as in Experiment 1 we found no evidence for any differences by trial type in this regard (all $B F s_{10}<0.66$ ).

Second round response times. We again analyzed second round choice response times split by trial type and choice consistency. As in Experiment 1, a pattern emerged where for control trials (consistent: $M=6.4 \mathrm{~s}, S D=6.8$, inconsistent: $M=14.5 \mathrm{~s}, S D=12.4$ ) and corrected trials (consistent: $M=8.0 \mathrm{~s}, S D=7.6$, inconsistent: $M=16.0 \mathrm{~s}, S D=13.9$ ) participants were considerably slower on inconsistent trials than on consistent trials (see Fig 3B). By contrast on accepted trials participants had similar choice response times when they were consistent $(M=9.7 \mathrm{~s}, S D=9.0)$ as when they were inconsistent $(M=9.5 \mathrm{~s}, S D=10.7)$.

We next tested these patterns using a mixed-effects regression on the log-transformed choice response times. The patterns of evidence were remarkably similar to those reported in the analysis of Experiment 1. We again found strong evidence, on average, for a difference in choice response times between consistent and inconsistent choices, such that inconsistent choices were on average slower than inconsistent choices $(b=0.44, S E=0.098, H D I=[0.25,0.64], p d=$ $\left.0.9999, B F_{10}=318\right)$. Similarly, there again were no differences in average choice response times between trial types (all $B F s_{10}<0.46$ ). We next examined contrasts between consistent and inconsistent choices separately for each trial type. As in Experiment 1, we found evidence for inconsistent choices being slower than consistent choices for both control trials (textitb $=0.83, S E=0.13, H D I=[0.56,1.09]$, $p d \approx 1.0, B F_{10}=2.39 * 10^{4}$ ) as well as for corrected trials (textitb $=0.54, S E=0.21, H D I=[0.11,0.95], p d=0.9942$, $\left.B F_{10}=6.15\right)$. Critically, for accepted trial we instead found evidence favoring the null of no difference (textitb $=-0.039$, $\left.S E=0.15, H D I=[-0.35,0.26], p d=0.60, B F_{10}=0.165\right)^{3}$. In sum, in Experiment 2, as in Experiment 1, for control and corrected trials there is a choice response time difference between consistent and inconsistent choices, while for accepted trials there is no such difference.

\section{Summary Experiment 2}

Pairs of participants made mutual decisions in the roommate selection task and received false feedback about which person they had selected on some trials. Remarkably, despite being two individuals monitoring the choice feedback, and despite the explicit and discussion based nature of the prior choices, we found that participants accepted the false feedback in the majority of trials. This extends the choice blindness paradigm beyond the study of intrapersonal decision processes to an interpersonal context. The observed correction rates matched those which would be expected if two independent individual observers corrected the false feedback, indicating no facilitation or additive effects from the group setting.

Our primary aim was to investigate choice-induced preference change in groups. As hypothesized, our results showed that accepting the choice blindness manipulation led to the group preference shifting. These findings are, to the best of our knowledge, the first demonstration of choice-induced preference change at the group level. As with individuals, analysis of the second round choice response times strengthened our interpretation that the inconsistent choices in the accepted manipulated trials reflects processes related to the preference change seen in the dyad. We again observed large differences in choice response times between consistent and inconsistent choices for control and corrected manipulated trials and none such for the accepted manipulated trials. Importantly, we found the same patterns of consistency and choice response times in individuals (Experiment 1). Together, these patterns in our data corroborate the interpretation that accepting the manipulation leads to a preference

\footnotetext{
${ }^{3} B F_{01}=6.06$
} 


\section{Experiment 2: Dyads}
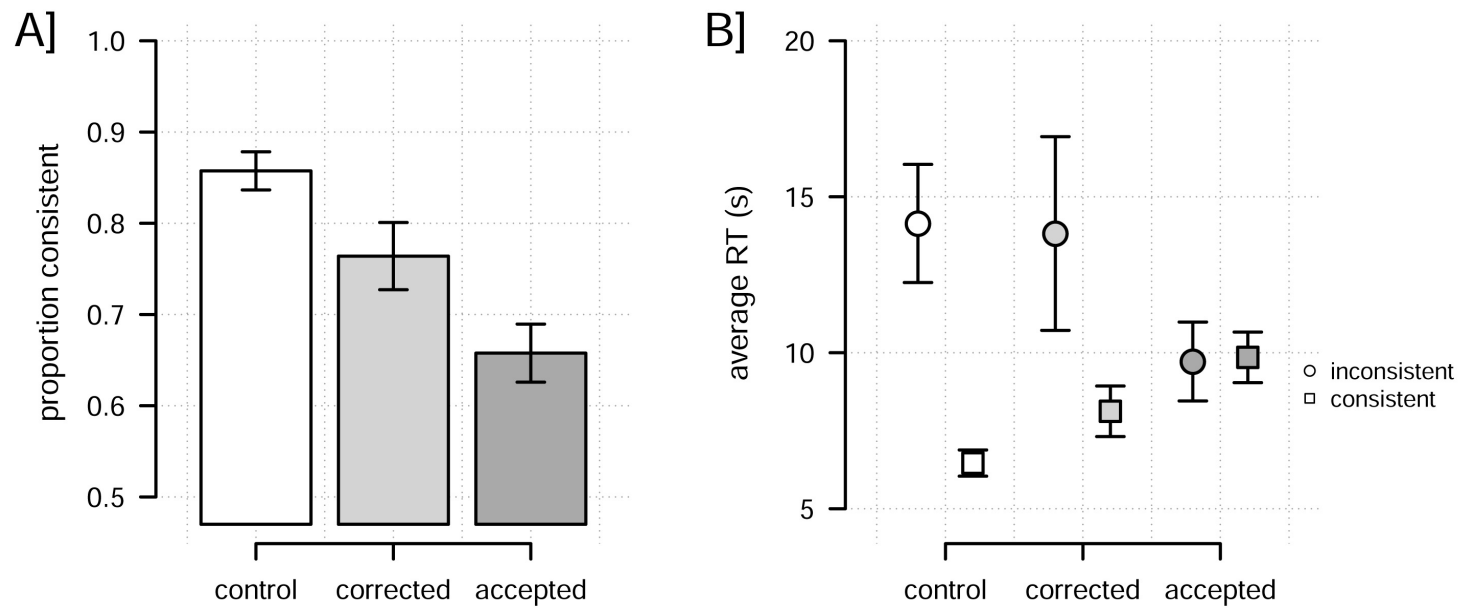

Figure 3. A] Proportion second round choices consistent with first round choices in Experiment 2. Error bars indicate standard errors. B] Average choice response times for second round choices in Experiment 2, split by consistency with first round choices. Circles represent inconsistent choices and squares consistent choices. Error bars indicate standard errors.

shift in the dyad. We return in the general discussion to the question of how to accommodate our findings in current explanations of choice-induced preference change and beyond.

\section{Experiment 3: From groups back to individuals}

An outstanding question from Experiment 2 concerns what happens to the manipulated preference when individuals leave the dyad. Do they keep the constructed preference from the group? Or do they display a different choice behavior, reflecting their own individual preferences? In Experiment 3 we tested this by again manipulating the choices of dyads in the roommate selection task but then having the participants make the second round choices individually.

\section{Method}

Participants. A total of 80 participants (40 dyads) were recruited from the student population of Lund University. Average age was $22.6(S D=2.6) .29$ participants were male. 15 dyads were mixed, 18 were female only, and 7 male only. Sample size was set so the number of participants (unit of analysis for the preference change) would match that of Experiments $1 \& 2$. All participants received a cinema voucher for participation. Dyads were formed by participants signing up to timeslots we made available through an online booking system. Before participating, we vetted the dyads to make sure that members were not acquainted with each other. Experiment 3 was approved by the Ethics Committee of Lund University (D.nr. 2016-1046).

Procedure. The first phase of the experiment was identical to that of Experiment 2. At the start of the second round of choices each dyad was split up and each participant sat at separate terminals. They then completed the second round on their own without communicating with each other.

\section{Results}

Correction of manipulation. Of the 160 M-trials across the 40 dyads, 61 (39\%) were corrected, 97 (61\%) were accepted and 2 trials were not classified due to equipment malfunction. $11(28 \%)$ dyads failed to make any correction, while on average each group made 1.54 detections $(S D=$ 1.31).

Choice consistency. We defined individual preference consistency as congruence between the dyad's first choice and the individual's second choice. Participants made second round choices consistent with their original decision in $75.3 \%$ of control trials. For corrected manipulated trials consistency was similar, $77.0 \%$ as was it for accepted manipulated trials $70.1 \%$, see Fig 4A. We analyzed second round choices using mixed-effects logistic regression, taking control trials as the reference level. Since each individual in the dyads give their confidence following the mutual choice, we could adjust for individual confidence in the analyses.

Unlike in Experiments 1 and 2, we found weak evidence in favor of the point null for differences between all three trial types (all $B F s_{10}<0.33$ ). Instead, we found a strong effect of confidence in the first round choice predicting consistency $(b$ $=0.80, S E=0.17, H D I=[0.45,1.15], p d=0.9999, B F_{10}$ $=440)$. Again, there were no difference by trial type in the effect of confidence on consistency (all $B F s_{10}<0.37$ ).

Second round response times. Finally we analyzed second round choice response times split by trial type and choice consistency. Like the the previous experiments, inconsistent choice response times were longer for control tri- 


\section{Experiment 3: Dyads to Individuals}
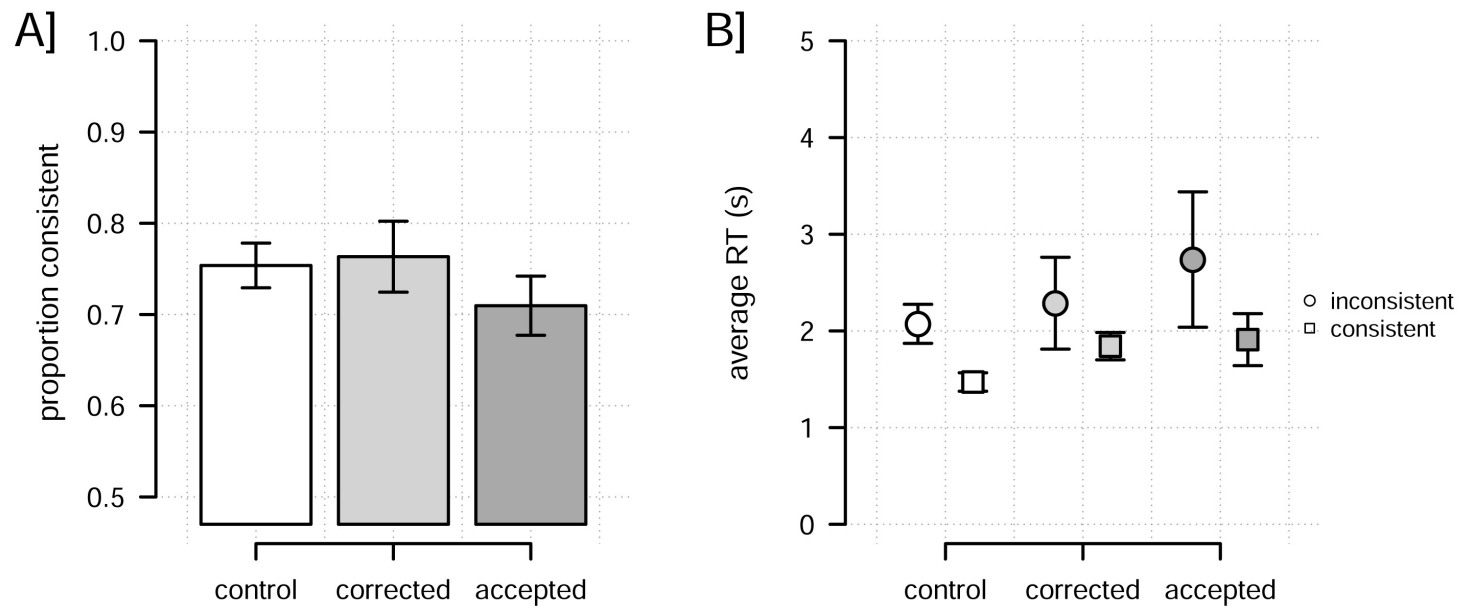

Figure 4. A] Proportion second round choices consistent with first round choices in Experiment 3. Error bars indicate standard errors. B] Average choice response times for second round choices in Experiment 3, split by consistency with first round choices. Circles represent inconsistent choices and squares consistent choices. Error bars indicate standard errors.

als (consistent: $M=1.5 \mathrm{~s}, S D=1.5$, inconsistent: $M=2.1 \mathrm{~s}$, $S D=1.8$ ), and corrected trials (consistent: $M=1.7 \mathrm{~s}, S D=$ 1.4, inconsistent: $M=2.4 \mathrm{~s}, S D=2.5)$. However, unlike the previous experiments, the same pattern was seen for accepted trials (consistent: $M=2.0 \mathrm{~s}, S D=3.1$, inconsistent: $M=2.8 \mathrm{~s}$, $S D=5.3$ ), see also Fig 4B.

Analyzing the data using a mixed-effects regression on the log-transformed choice response times indicated weak evidence for a difference in choice response times between consistent and inconsistent choices, such that inconsistent choices were on average slower than inconsistent choices $(b$ $=0.20, S E=0.064, H D I=[0.083,0.33], p d=0.9985, B F_{10}$ $=8.90)$. Again, there were no differences in average choice response times between trial types (all $B F s_{10}<0.16$ ). We found weak evidence for a difference between response times when making consistent and inconsistent choices in the control trials (textitb $=0.28, S E=0.10, H D I=[0.079,0.48]$, $p d=0.9960, B F_{10}=5.12$ ), but not in the corrected (textitb $=0.18, S E=0.14, H D I=[-0.10,0.46], p d=0.90, B F_{10}=$ 0.37 ) or in the accepted trials (textitb $=0.15, S E=0.12, H D I$ $\left.=[-0.079,0.38], p d=0.9045, B F_{10}=0.30\right)$.

\section{Summary Experiment 3}

We replicated our findings of choice blindness in dyads from Experiment 2, as the dyads accepted the false feedback at similar levels as previously found and thus again demonstrating that the choice blindness paradigm extends to dyadic decision making. Novel in Experiment 3 was that the second round choices were done by the individual dyad members separately. In this case we found no effect of the choice blindness manipulation on choice consistency. In other words, the choice-induced preference change stem- ming from manipulating the dyad's belief in their own past choice did not transfer to individual preferences. The second round choice response times corroborated this interpretation as we, unlike in the previous experiments, found small or no differences in the response time patterns between control, correct and accepted trials depending on choice consistency.

\section{General Discussion}

We investigated if choice-induced preference change is found in groups by giving dyads false feedback about mutual decisions using the choice blindness paradigm. To do so, we introduced a novel choice task - the roommate selection task - in which dyads are presented with two pictures of faces and have to decide which of the two people they prefer to share an apartment with. Using this task, we demonstrated that dyads are susceptible to choice blindness manipulations as they often both accept and argue for a choice they were led to believe they had made. Importantly, we found that dyads when accepting the false feedback about their decisions were more likely to select the believed to have been chosen option in a second round of choices, thereby also exhibiting choiceinduced preference change. Finally, we found that individuals, once separated from the dyad, did not exhibit the same preference change as measured in subsequent choices.

\section{Choice blindness in groups}

The first contribution of this study is to demonstrate that the choice blindness paradigm can be used to study decision making in a group setting. The correction rate was notably higher in dyads than for the individual participants in Experiment 1. However, it was not higher than what would 
be expected if two independent observers had aggregated their scores, suggesting no additive effects from group membership. This result is in contrast to findings from related areas, in which groups systematically outperform the individual (Bahrami et al., 2010; Knott et al., 2007; Malcolmson et al., 2007b; Tindale \& Winget, 2019). These results are promising for scaling choice blindness manipulations to larger groups, which could allow for novel ways of studying the impact of false feedback and erroneous beliefs in social settings. Moreover, these results suggest that it should be possible to investigate how groups perform in other tasks where choice blindness has been used, for example to study political attitudes (Strandberg et al., 2018), eye-witness testimony (Sagana et al., 2014) or financial decisions (McLaughlin \& Somerville, 2013).

Choice blindness was originally introduced as a methodology to study self-knowledge in individual decision making, with a focus on the verbal reports of the participants and comparisons between manipulated and non-manipulated trials (Johansson et al., 2005; Johansson, Hall, Sikström, Tärning, \& Lind, 2006). In this context, it is a puzzle as to why people would rationalize choices they never made and what function this type of confabulation could serve. It has recently been argued that rationalization functions to aid communication between cognitively encapsulated behavioral controllers, for example informing a conscious decision system about the preferences of a habitual system (Cushman, 2020). We have proposed that a primary function of rationalization rather is to smooth interpersonal communication and facilitate coordination necessary for joint action (Pärnamets, Johansson, \& Hall, 2020). The experiments described here demonstrate collaborative confabulation - rationalization in the service of representational exchange (Cushman, 2020) between two decision makers. This is then another path of exploration opened by the current results, one that may connect to prior work on memory reshaping following conversations (Hirst \& Echterhoff, 2012).

\section{Choice-induced preference change in groups}

Our second main contribution is that in Experiment 2 we demonstrated the first experimental evidence of choiceinduced preference change for group rather than individual choices. Dyads were more likely to change their preferences during the second round of choices as a result of accepting false feedback about which option they had previously chosen. The effects of accepting the false feedback were also evident in the time it took for the dyads to reach a decision when making the choices the second time. In control trials, the second round choice response times of inconsistent choices are drastically higher than those of consistent choices, presumably due to an increased cognitive effort reversing a previously held position. But when dyads reverse their initial preferences as a result of accepting the false feed- back, they take the same amount of time to reach that decision as when making a decision consistent with their original choice. This indicates that the change in preference observed in choice behavior has become an integrated part of the joint preferences of the dyads. Having thus established choiceinduced preference change in groups, we next turn to how to interpret these results in light of extant theories of preference change.

Cognitive dissonance. In the standard interpretation of choice-induced preference change, individuals alter their preferences in line with the choice to reduce cognitive dissonance arising from having entertained positive aspects of the rejected option and negative aspects of the chosen option during deliberation (Brehm, 1956; Festinger, 1962; Harmon-Jones \& Mills, 2019). This post-choice reevaluation is thought to take place immediately following the choice, since the experienced dissonance would be the largest when the just rejected option is still salient to the decision-maker. Given the structure of the choice blindness paradigm, this type of explanation does not seem to fit the preference change seen in Experiments 1 and 2, or previous work on choice blindness and preference change (Johansson et al., 2014; Luo \& Yu, 2017; Strandberg et al., 2018; Taya et al., 2014). Whatever drives the change, it can not be the relationship between the deliberation and the actual - originally intended - outcome of the choice, since this would predict a shift in preference in the opposite direction of what we observe. Instead, preference change must arise in relation to the presented option, conditional on its acceptance, during the false-feedback portion of the experimental trial.

According to the basic definition of cognitive dissonance theory, dissonance arises whenever two conflicting cognitions are held (Festinger, 1962). To accommodate the results of the experiments here within the dissonance framework, the moment of dissonance could be the presentation of the false feedback, with the memory of the actual choice and the prospect of accepting the manipulated outcome as the two cognitions in conflict. If this is the case, then when the dyad accepts the manipulated choice, both members respond to the dissonance this entails and alter their preferences in line with the manipulation. While prima facie reasonable, we believe that the lack of a preference effect in our Experiment 3 cautions against adopting this interpretation. If both individuals experience dissonance and adjust their preferences to escape it, then we should have expected this preference change to be expressed also in Experiment 3. Instead we do not find that accepting the manipulation alters preferences when dyad members make their second round choices individually. The situation is further complicated by the findings in the cognitive dissonance literature that group membership tends to shield members from dissonance (Cooper \& Stone, 2000; McKimmie, 2015), in which case the theory should not predict preference change in Experiment 2 either. While 
we agree with the general perspective of dissonance theory, i.e. that agents seek consistency or coherence in their cognitions (see also Hornsby \& Love, 2020), as far as we can tell, the theory is currently under-specified to fully explain the experimental results presented here. As such, this study presents a challenge for cognitive dissonance explanations of choice-induced preference change.

The role of memory. Several recent studies have suggested explaining choice-induced preference change directly with reference to memory processes (Chammat et al., 2017; Salti, El Karoui, Maillet, \& Naccache, 2014; Voigt, Murawski, Speer, \& Bode, 2019). Generally, people remember items better if they are given a choice, or even the illusion of choice (Murty, DuBrow, \& Davachi, 2015). In line with this, choice-induced preference change has been shown to correlate with increased activity in dorsolateral prefrontal cortex during choice (Voigt et al., 2019), but only for items that are remembered post-choice (Chammat et al., 2017; Salti et al., 2014; Voigt et al., 2019). Memory for past chosen items has additionally been show to correlate with hippocampal activation, but only for accurately remembered items in a rate-choice-rate preference change condition (Chammat et al., 2017; Voigt et al., 2019). Recent research has found that preferences are updated during the act of choosing (Voigt et al., 2019), indicating that memory consolidation mechanisms endogenous to the choice process play part in explaining choice-induced preference change, in line with recent computational modeling of behavioral data (Hornsby \& Love, 2020; Lee \& Daunizeau, 2019).

Whatever merits this perspective on choice-induced preference change brings with it, it too has trouble accounting for choice blindness and the consequent effects of accepting false feedback about choices. First of all, if chosen items are remembered better, this makes choice blindness in both individuals and dyads even more remarkable, as it would increase the probability of detection of the manipulated feedback (Lejarraga et al., 2014; Olsson et al., 2006). Secondly, iterating the argument presented above in relation to cognitive dissonance, the choice blindness manipulation lead to a preference change in the opposite direction to what memorybased theories predict. The false feedback occurs several seconds following the original choice, it implies that regardless of the plausibility of choice-induced preference change stemming partly from cognitive processes operating at the moment of choice, the effects we obtained must arise from processes taking place after the original choice has been made. This interpretation accords with research suggesting that hippocampal correlates of choice-induced preference change reflect consistency effects occurring during the remembering psot-choice (Chammat et al., 2017; Salti et al., 2014).

Collaboratively confabulating shared preferences. Choice-induced preference change can also be explained by reference to processes of self-perception (Bem, 1972).
While self-perception theory and cognitive dissonance theory are notoriously difficult to separate, we have previously argued that choice blindness lends itself towards interpretation through the lens of self-perception theory when it comes to individual choices (Johansson et al., 2014; Strandberg et al., 2018). But how should the "self" in self-perception be understood in the context of the current result, when it is a dyad shifting it's preference? And how should we interpret the results of Experiment 3, when the change in dyadic preference did not translate to the individual level?

We suggest that our pattern of results indicate that the dyads construct a shared preference that is adhered to in the dynamic of their follow-up decisions. This mutual preference is affected by the mutual perception of what the dyad's past preference was. However, when removed from the context of the mutual choice the belief about what the dyad choose no longer binds the individual. This is akin to the classic findings in the study of decision making that changing the mode of elicitation - how and what questions are asked will change the preference, value or attitude expressed (Fischhoff, Slovic, \& Lichtenstein, 1988). We know that in individual decision making, acceptance of false feedback about past choices alters the memory of those choices (Cochran et al., 2016; Henkel \& Mather, 2007; Pärnamets et al., 2015). But in the dyad, the individual level memories and beliefs must be negotiated and agreed upon when the two participants jointly accept and explain the manipulated choice.

The response time for the second round choices also support this interpretation. When the individuals in Experiment 1 and the dyads in Experiment 2 reverse their initial preferences after having accepted the false feedback, they take the same amount of time to reach a decision as when making a decision consistent with the original choice. But when we look at trials with individual preference change in Experiment 3 , the participants take significantly longer time to reach a decision compared to trials in which they stay consistent with the original choice. This indicates that not only is less preference change at the individual level in Experiment 3, but that when it occurs it is not accompanied with the same cognitive ease observed in Experiment 1 and 2. Taken together, our findings provide a demonstration of the separability of the preferences of the individual and preferences of the individual as a member of a group following a group decision.

In sum, choice-induced preference change as a phenomenon generalizes to groups and it does so through affecting shared representations of the group's preferences. If this wasn't the case, and group decisions merely aggregated individual preferences, our patterns of results would not be obtained. Social interactions add an additional domain to how we how we interpret or infer our own behaviour, an extra domain for which the brain must account for in its model of the world. The putative computational substrates of how 
these shared domains are formed or inferred and modulated in the choice blindness paradigm is an area we wish to explore in the future (Bolis \& Schilbach, 2020; Kelly, Kriznik, Kinmonth, \& Fletcher, 2019; Ramstead, Veissière, \& Kirmayer, 2016).

Preference change in choice blindness. Our findings follow and build on a growing number of similar results showing that accepting false feedback about past choices influence future choices and behaviors (Johansson et al., 2014; Luo \& Yu, 2017; Strandberg et al., 2018; Taya et al., 2014). Recently, Bortolotti and Sullivan-Bissett (2019), suggested two interpretations of the general choice blindness effect. They contrast a 'choice error' interpretation whereby choice blindness reveals that people can be mistaken about their previous choices, with a 'choice change' interpretation whereby choice blindness reveals that people can be manipulated into reversing their choices within minutes of first making them. In their preferred second alternative, Bortolotti and SullivanBissett argue that when defending and explaining a manipulated choice, the participants should be understood as actually changing their choice, by aligning their preferences with the reasons they produce. The results of the present study support this interpretation, both in individuals (Experiment 1) and in dyads (Experiment 2). We see a significant preference change in line with the accepted manipulation, and we see that subsequent choice deliberation is influenced, as measured in choice response times. We have previously found that the memory of the choice is influenced by false feedback manipulation (Johansson, Hall, \& Sikström, 2008; Pärnamets et al., 2015), and we have every reason to believe that a similar result would have been found here. This suggests that, when the manipulation is accepted, the preference actually changes and becomes the new psychological reality of the participants. We have previously argued (Strandberg et al., 2018) that preference change in choice-blindness reflects a rational response to an actual change in beliefs about past choices (see also Luo \& Yu, 2017; Pärnamets et al., 2015); it appears to us that the "choice change" interpretation of Bortolotti and Sullivan-Bissett can be naturally aligned with that position.

\section{Limitations and future directions}

Our study is not without limitations. Chief of these is that in the present experiments we limit our study to dyads (Moreland, 2010). There is likely an upper limit of number of participants monitoring the same mutual choice blindness decision before it no longer can be performed unnoticed; however, with increasing group sizes come increasing social and normative pressures to conform to the group which may counteract the effects of pure aggregation of monitoring individuals (Bond, 2005; Raafat et al., 2009). Utilizing choice blindness in larger groups would provide other important research opportunities. For example, in larger groups, selec- tively giving only some participants false feedback may provide important avenues to perturb perceived group consensus and an effective avenue for testing theories of deliberation and norm following. As group decision making is critical in for example legal settings such experiments may prove of considerable societal value.

In future studies we aim to further elucidate how this element of "collaborative confabulation" compares to individual rationalizations, and how it might relate to the direction and magnitude of preference change in groups. For example, in (Strandberg et al., 2018) we found that for political attitudes, the attitude change observed following a choice blindness manipulation was increased as a factor of how much the participants had discussed and verbalised their reasons for the accepted manipulated choice. This pattern of results would be of high interest to explore in a group setting. Another key outstanding question is to understand when a mutual preference acquires binding force to the constituent individuals. For example, according to shared reality theory (Echterhoff, Higgins, \& Levine, 2009; Rossignac-Milon \& Higgins, 2018), cognitions arrived at interpersonally should have a stronger force than non-socially acquired cognitions. By manipulating participants motivations together with their beliefs about their past choices, key predictions from shared reality theory could be tested to deepen our understanding of how shared preferences are constructed and promulgated back to constituent individuals.

Above we discussed the many findings linking simple acts of choosing to enhanced memory (Murty et al., 2015) and to choice-induced preference change in individuals (Chammat et al., 2017; Salti et al., 2014). An important topic for future research would be to link the dyadic effects observed here back to the neural level, using fMRI hyperscanning techniques. Doing so can help arbitrate explanations for what happens at the individual level, as well as paint a better picture for how group choices are represented and processed.

In the present study we allowed dyads to freely negotiate their individual preferences; in future work, by modelling natural social roles, for example where one member is given a leadership position or social status is otherwise manipulated, we can gain further insights into preference formation. A more applied example would be to use choice blindness to study collaborative search procedures, such as those followed during security screening at airports (cf. Malcolmson et al., 2007a, 2007b).

\section{Conclusion}

Many of life's important decisions are made in pairs or groups - from where to meet for a first coffee to picking a wedding venue; from replacing a flat-mate to appointing a new head of department. Here we showed that dyads will accept false feedback about their past choices and that doing so affects their later choices. This result extends the 
phenomenon of choice-induced preference change to groups, and reminds us that in this hyper-connected social media age to be ever vigilant of the accuracy of the group feedback that we receive (Allington, Duffy, Wessely, Dhavan, \& Rubin, 2020; Van Bavel et al., 2020).

\section{References}

Allington, D., Duffy, B., Wessely, S., Dhavan, N., \& Rubin, J. (2020). Health-protective behaviour, social media usage and conspiracy belief during the covid-19 public health emergency. Psychological medicine, 1-7.

Bahrami, B., Olsen, K., Latham, P. E., Roepstorff, A., Rees, G., \& Frith, C. D. (2010). Optimally interacting minds. Science, 329(5995), 1081-1085.

Bem, D. J. (1972). Self-perception theory. In Advances in experimental social psychology (Vol. 6, pp. 1-62). Elsevier.

Bolis, D., \& Schilbach, L. (2020). 'i interact therefore i am': The self as a historical product of dialectical attunement. Topoi, 39(3), 521-534.

Bond, R. (2005). Group size and conformity. Group processes $\mathcal{E}$ intergroup relations, 8(4), 331-354.

Bortolotti, L., \& Sullivan-Bissett, E. (2019). Is choice blindness a case of self-ignorance? Synthese, 1-18.

Brehm, J. W. (1956). Postdecision changes in the desirability of alternatives. The Journal of Abnormal and Social Psychology, 52(3), 384

Brennan, S. E., Chen, X., Dickinson, C. A., Neider, M. B., \& Zelinsky, G. J. (2008). Coordinating cognition: The costs and benefits of shared gaze during collaborative search. Cognition, 106(3), 1465-1477.

Bürkner, P.-C., et al. (2017). brms: An r package for bayesian multilevel models using stan. Journal of Statistical Software, 80(1), 1-28.

Chammat, M., El Karoui, I., Allali, S., Hagège, J., Lehongre, K., Hasboun, D., ... others (2017). Cognitive dissonance resolution depends on episodic memory. Scientific reports, 7 , 41320.

Chen, M. K., \& Risen, J. L. (2010). How choice affects and reflects preferences: revisiting the free-choice paradigm. Journal of personality and social psychology, 99(4), 573.

Cheung, T., Junghans, A., Dijksterhuis, G. B., Kroese, F., Johansson, P., Hall, L., \& De Ridder, D. (2016). Consumers' choice-blindness to ingredient information. Appetite, 106, 2-12.

Cialdini, R. B., \& Goldstein, N. J. (2004). Social influence: Compliance and conformity. Annual Review of Psychology, 55, 591-621.

Cochran, K. J., Greenspan, R. L., Bogart, D. F., \& Loftus, E. F. (2016). Memory blindness: Altered memory reports lead to distortion in eyewitness memory. Memory $\mathcal{E}$ Cognition, 44(5), 717-726.

Cooper, J., \& Stone, J. (2000). Cognitive dissonance and the social group. In D. J. Terry \& M. A. Hogg (Eds.), Cognitive dissonance: Reexamining a pivotal theory in psychology (pp. 227-244). Lawrence Erlbaum Associates Publishers.

Cushman, F. (2020). Rationalization is rational. Behavioral and Brain Sciences, 43, e28. doi: 10.1017/
S0140525X19001730

Echterhoff, G., Higgins, E. T., \& Levine, J. M. (2009). Shared reality: Experiencing commonality with others' inner states about the world. Perspectives on Psychological Science, 4(5), 496-521.

Edwards, W. (1954). The theory of decision making. Psychological bulletin, 51(4), 380

Egan, L. C., Bloom, P., \& Santos, L. R. (2010). Choice-induced preferences in the absence of choice: Evidence from a blind two choice paradigm with young children and capuchin monkeys. Journal of Experimental Social Psychology, 46(1), 204-207.

Festinger, L. (1962). A theory of cognitive dissonance (Vol. 2). Stanford university press.

Fischhoff, B., Slovic, P., \& Lichtenstein, S. (1988). Knowing what you want: Measuring labile values. In D. Bell, H. Raiffa, \& A. Tversky (Eds.), Decision making: Descriptive, normative, and prescriptive interactions (pp. 98-421). Cambridge University Press.

Gerard, H. B., \& White, G. L. (1983). Post-decisional reevaluation of choice alternatives. Personality and Social Psychology Bulletin, 9(3), 365-369.

Hall, L., Johansson, P., \& Strandberg, T. (2012). Lifting the veil of morality: Choice blindness and attitude reversals on a selftransforming survey. PloS one, 7(9), e45457.

Harmon-Jones, E., \& Mills, J. (2019). An introduction to cognitive dissonance theory and an overview of current perspectives on the theory. In E. Harmon-Jones (Ed.), Cognitive dissonance: Reexamining a pivotal theory in psychology (pp. 324). American Psychological Association.

Henkel, L. A., \& Mather, M. (2007). Memory attributions for choices: How beliefs shape our memories. Journal of Memory and Language, 57(2), 163-176.

Hirst, W., \& Echterhoff, G. (2012). Remembering in conversations: The social sharing and reshaping of memories. Annual review of psychology, 63.

Hornsby, A. N., \& Love, B. C. (2020). How decisions and the desire for coherency shape subjective preferences over time. Cognition, 200, 104244.

Ingham, A. G., Levinger, G., Graves, J., \& Peckham, V. (1974). The ringelmann effect: Studies of group size and group performance. Journal of experimental social psychology, 10(4), 371-384

Izuma, K., Akula, S., Murayama, K., Wu, D.-A., Iacoboni, M., \& Adolphs, R. (2015). A causal role for posterior medial frontal cortex in choice-induced preference change. Journal of Neuroscience, 35(8), 3598-3606.

Izuma, K., \& Murayama, K. (2013). Choice-induced preference change in the free-choice paradigm: a critical methodological review. Frontiers in psychology, 4, 41.

Johansson, P., Hall, L., \& Sikström, S. (2008). From change blindness to choice blindness. Psychologia, 51(2), 142-155.

Johansson, P., Hall, L., Sikström, S., \& Olsson, A. (2005). Failure to detect mismatches between intention and outcome in a simple decision task. Science, 310(5745), 116-119.

Johansson, P., Hall, L., Sikström, S., Tärning, B., \& Lind, A. (2006). How something can be said about telling more than we can know: On choice blindness and introspection. Con- 
sciousness and cognition, 15(4), 673-692.

Johansson, P., Hall, L., Tärning, B., Sikström, S., \& Chater, N. (2014). Choice blindness and preference change: You will like this paper better if you (believe you) chose to read it! Journal of Behavioral Decision Making, 27(3), 281-289.

Kelly, M. P., Kriznik, N. M., Kinmonth, A. L., \& Fletcher, P. C. (2019). The brain, self and society: a social-neuroscience model of predictive processing. Social neuroscience, 14(3), 266-276.

Kerr, N. L., \& Tindale, R. S. (2004). Group performance and decision making. Annual Review of Psychology, 55, 623-655.

Knott, C. C., Nelson, W. T., McCroskey, M. K., \& Miller, B. T. (2007). Do you see what i see? effects of communication on scanning strategies in change detection by individuals and teams of observers. In Proceedings of the human factors and ergonomics society annual meeting (Vol. 51, pp. 11861190).

Konovalov, A., \& Krajbich, I. (2019). Revealed strength of preference: Inference from response times. Judgment $\mathcal{E}$ Decision Making, 14(4).

Kugler, T., Kausel, E. E., \& Kocher, M. G. (2012). Are groups more rational than individuals? a review of interactive decision making in groups. Wiley Interdisciplinary Reviews: Cognitive Science, 3(4), 471-482.

Lee, D., \& Daunizeau, J. (2019). Choosing what we like vs liking what we choose: How choice-induced preference change might actually be instrumental to decision-making. bioRxiv, 661116.

Lejarraga, T., Lejarraga, J., \& Gonzalez, C. (2014). Decisions from experience: How groups and individuals adapt to change. Memory E cognition, 42(8), 1384-1397.

Link, S. W. (1975). The relative judgment theory of two choice response time. Journal of Mathematical Psychology, 12(1), 114-135.

Luo, J., \& Yu, R. (2017). The spreading of alternatives: Is it the perceived choice or actual choice that changes our preference? Journal of Behavioral Decision Making, 30(2), 484-491.

Makowski, D., Ben-Shachar, M. S., \& Lüdecke, D. (2019). bayestestr: Describing effects and their uncertainty, existence and significance within the bayesian framework. Journal of Open Source Software, 4(40), 1541.

Malcolmson, K. A., Reynolds, M. G., \& Smilek, D. (2007a). Collaboration during visual search. Psychonomic bulletin $\mathcal{E}$ review, 14(4), 704-709.

Malcolmson, K. A., Reynolds, M. G., \& Smilek, D. (2007b). Collaborative search in real-world scenarios. Journal of Vision, 7(9), 724-724.

McKimmie, B. M. (2015). Cognitive dissonance in groups. Social and Personality Psychology Compass, 9(4), 202-212.

McLaughlin, O., \& Somerville, J. (2013). Choice blindness in financial decision making. Judgment and Decision Making, $8(5), 577$.

Moreland, R. L. (2010). Are dyads really groups? Small Group Research, 41(2), 251-267.

Murty, V. P., DuBrow, S., \& Davachi, L. (2015). The simple act of choosing influences declarative memory. Journal of Neuroscience, 35(16), 6255-6264.

Olsson, A.-C., Juslin, P., \& Olsson, H. (2006). Individuals and dyads in a multiple-cue judgment task: Cognitive processes and performance. Journal of Experimental Social Psychology, 42(1), 40-56.

Pärnamets, P., Hall, L., \& Johansson, P. (2015). Memory distorions resulting from a choice blindness task. In D. C. Noelle et al. (Eds.), 37th annual conference of the cognitive science society: Mind, technology, and society (pp. 1823-1828). Cognitive Science Society.

Pärnamets, P., Johansson, P., \& Hall, L. (2020). Letting rationalizations out of the box. Behavioral and Brain Sciences, 43 , e41. doi: 10.1017/S0140525X1900219X

Peirce, J. W. (2007). Psychopy—psychophysics software in python. Journal of neuroscience methods, 162(1-2), 8-13.

Raafat, R. M., Chater, N., \& Frith, C. (2009). Herding in humans. Trends in cognitive sciences, 13(10), 420-428.

Ramstead, M. J., Veissière, S. P., \& Kirmayer, L. J. (2016). Cultural affordances: Scaffolding local worlds through shared intentionality and regimes of attention. Frontiers in psychology, 7, 1090 .

Ratcliff, R., \& Rouder, J. N. (1998). Modeling response times for two-choice decisions. Psychological science, 9(5), 347356.

Rossignac-Milon, M., \& Higgins, E. T. (2018). Beyond intrapersonal cognitive consistency: shared reality and the interpersonal motivation for truth. Psychological Inquiry, 29(2), 86-93.

Sagana, A., Sauerland, M., \& Merckelbach, H. (2014). 'this is the person you selected': Eyewitnesses' blindness for their own facial recognition decisions. Applied Cognitive Psychology, 28(5), 753-764.

Salti, M., El Karoui, I., Maillet, M., \& Naccache, L. (2014). Cognitive dissonance resolution is related to episodic memory. PloS one, 9(9).

Shteynberg, G. (2015). Shared attention. Perspectives on Psychological Science, 10(5), 579-590.

Slovic, P., Fischhoff, B., \& Lichtenstein, S. (1977). Behavioral decision theory. Annual review of psychology, 28(1), 1-39.

Strandberg, T., Sivén, D., Hall, L., Johansson, P., \& Pärnamets, P. (2018). False beliefs and confabulation can lead to lasting changes in political attitudes. Journal of Experimental Psychology: General, 147(9), 1382.

Taya, F., Gupta, S., Farber, I., \& Mullette-Gillman, O. A. (2014). Manipulation detection and preference alterations in a choice blindness paradigm. PloS one, 9(9).

Tindale, R. S., \& Winget, J. R. (2019). Group decision-making. In Oxford research encyclopedia of psychology. Oxford University Press.

Tyebjee, T. T. (1979). Response time, conflict, and involvement in brand choice. Journal of Consumer Research, 6(3), 295304.

Van Bavel, J. J., Harris, E. A., Pärnamets, P., Rathje, S., Doell, K., \& Tucker, J. A. (2020). Political psychology in the digital (mis) information age.

Voigt, K., Murawski, C., Speer, S., \& Bode, S. (2019). Hard decisions shape the neural coding of preferences. Journal of Neuroscience, 39(4), 718-726.

Weldon, E., \& Gargano, G. M. (1985). Cognitive effort in additive task groups: The effects of shared responsibility on the 
quality of multiattribute judgments. Organizational Behavior and Human Decision Processes, 36(3), 348-361. 